Pacific Journal of Mathematic 


\title{
A NOTE ON THE DIMENSION THEORY OF RINGS
}

\author{
A. SEIDENBERG
}

1. Introduction. Let $O$ be an integral domain. If in $O$ there is a proper chain

$$
(0) \subset P_{1} \subset P_{2} \subset \cdots \subset P_{n} \subset(1)
$$

of prime ideals, but no such chain

$$
(0) \subset P_{1}^{\prime} \subset \cdots \subset P_{n+1}^{\prime} \subset(1),
$$

then $O$ will be said to be $n$-dimensional. Let $O$ be of dimension $n$ : the question is whether the polynomial ring $O[x]$ is necessarily $(n+1)$-dimensional. Here, as throughout, $x$ is an indeterminate.

By an $F$-ring we shall mean a l-dimensional ring $O$ such that $O[x]$ is not 2dimensional (i. e., the proposed assertion that $O[x]$ is necessarily 2-dimensional fails). Given an $F$-ring, we try by definite constructions to pass to a larger $F$ ring having the same quotient field: this restricts the class of rings in which to look for an $F$-ring-a priori we do not know they exist. In this way we also come (in Theorem 8 below) to a complete characterization of $F$-rings: if $O$ is 1 -dimensional, then $O[x]$ is 2-dimensional if and only if every quotient ring of $\bar{O}$, the integral closure of $O$, is a valuation ring. The rings $\bar{O}$ thus coincide (for dimension 1) with Krull's Multiplikationsringe [ 5; p. 554].

2. Preliminary results. The first five theorems are of a preparatory character, and the proofs offer no difficulties.

THEOREM 1. Let $O$ be an arbitrary commutative ring with $1, P_{1}, P_{2}, P_{3}$ distinct ideals in $O[x]$. If $P_{1} \subset P_{2} \subset P_{3}$, and $P_{2}$ and $P_{3}$ are prime ideals, then $P_{1}$, ${ }_{2}, P_{3}$ cannot have the same contraction to $O$.

Proof. Let

$$
P_{1} \cap O=P_{2} \cap O=p \text {, }
$$

Received May 15, 1952.

Pacific J. Math. 3 (1953), 505-512 
and consider

$$
O[x] / P_{2}=\bar{O}[\bar{x}]
$$

where $\bar{x}$ is the residue of $x$ and $\bar{O} \simeq O / p$. Since

$$
\bar{O}[x] \cdot p \subseteq P_{1} \subset P_{2},
$$

$\bar{x}$ is algebraic over the integral domain $\bar{O}$. Let $\bar{P}_{3}$ be the image of $P_{3} ;$ then $\bar{P}_{3} \neq$ (0); but also $\bar{P}_{3} \cap \bar{O} \neq(0)$. In fact, let $\gamma \in \bar{P}_{3}, \gamma \neq 0$. Then

$$
c_{0} \gamma^{n}+c_{1} \gamma^{n-1}+\cdots+c_{n}=0
$$

for some $c_{i} \in \bar{O}, c_{n} \neq 0$; and $c_{n} \in \bar{P}_{3} \cap \bar{O}$. Hence also $P_{3} \cap O \neq p$,

Corollary. If $O$ is 1-dimensional, and $P_{1}, P_{2}, P_{3}$ are distinct prime ideals in $O[x]$ different from (0) with $P_{1} \subset P_{2} \subset P_{3}$, then $P_{1} \cap O=(0), P_{2}$ is the extension of its contraction to $O$, and $P_{3}$ is maximal.

Proof. If $P_{1} \cap O \neq(0)$, then $P_{1}, P_{2}, P_{3}$ would all have to contract to the same maximal ideal in $O$. So

$$
P_{1} \cap O=(0) \text { and } P_{2} \cap O=p \neq(0) .
$$

Were $O[x] \cdot p \subset P_{2}$ properly, then, since $O[x] \cdot p$ is prime,

$$
O[x] \cdot p \cap O=(0),
$$

whereas

$$
O[x] \cdot p \cap O=p .
$$

So $O[x] \cdot p=P_{2}$. Were $P_{3}$ not maximal, we would have $P_{2} \cap O=(0)$.

For the foregoing theorem, see also [4; Th. 10, p. 375] .

The OREm 2. If $O$ is n-dimensional, then $O[x]$ is at least $(n+1)$-dimensional and at most $(2 n+1)$-dimensional.

Proof. Let

$$
(0) \subset P_{1} \subset P_{2} \subset \cdots \subset P_{n} \subset(1)
$$

be a proper chain of prime ideals in $O$. Then

$$
(0) \subset O[x] \cdot P_{1} \subset O[x] \cdot P_{2} \subset \cdots \subset O[x] \cdot P_{n} \subset
$$


is also a proper chain of prime ideals in $O[x]$; and $O[x] \cdot P_{n}$ is not maximal, since, for example,

$$
O[x] \cdot P_{n} \subset\left(O[x] \cdot P_{n}, x\right) \subset(1) .
$$

(Here, as throughout, we use the symbol $\subset$ for proper inclusion.) Hence $O[x]$ is at least $(n+1)$-dimensional. Let now $O$ be $n$-dimensional, and consider a chain

$$
\text { (0) } \subset P_{1}^{\prime} \subset \cdots \subset P_{m}^{\prime} \subset
$$

of prime ideals in $O[x]$. Let there be $s$ distinct ideals among the contractions

$$
\text { (0) } \cap O, P_{1}^{\prime} \cap O, \cdots, P_{m}^{\prime} \cap O \text {. }
$$

Then

$$
m+1<2 s \leq 2(n+1) \text {, so } m \leq 2 n+1 \text {. }
$$

THEOREM 3. If $O$ is n-dimensional but $O[x]$ is not $(n+1)$-dimensional, then for at least one minimal prime ideal $p$ of $O$ either the quotient ring $O_{p}$ is an $F$-ring or $O / p$ is m-dimensional and $O / p[x]$ is not $(m+1)$-dimensional, and $m<n$.

Proof. Suppose that for some minimal prime ideal $p$ of $O, O[x] \cdot p$ is not minimal in $O[x]$; that is, there exists a prime ideal $P$ such that

$$
\text { (0) } \subset P \subset O[x] \cdot p \text {. }
$$

Then

$$
(0) \subset O_{p}[x] \cdot P \subset O_{p}[x] \cdot p
$$

is also a chain of prime ideals in $O_{p}[x]$, as one easily verifies. Since $O_{p}[x] \cdot p$ is not maximal, this shows that $O_{p}$ is an $F$-ring. We pass then to the case that $O[x] \cdot p$ is minimal for every minimal prime ideal $p$ of $O$. Let

$$
(0) \subset P_{1}^{\prime} \subset \cdots \subset P_{n+2}^{\prime} \subset(1)
$$

be a chain of prime ideals in $O[x]$. If

$$
P_{1}^{\prime} \cap O=p \neq(0),
$$

then $O / p$ is at most $(n-1)$-dimensional, and $O[x] / O[x] \cdot p$ is a polynomial ring in one variable over $O / p$ and is at least $(n+1)$-dimensional. So we must suppose 


$$
P_{1}^{\prime} \cap O=(0)
$$

but then

$$
P_{2}^{\prime} \cap O=p_{2} \neq(0)
$$

let $p$ be a minimal prime ideal contained in $p_{2}$ - such exists since $O$ is finite dimensional; then $O[x] \cdot p \subset P_{2}^{\prime}$, properly, since $O[x] \cdot p$ is minimal but $P_{2}^{\prime}$ is not. Replacing $P_{1}^{\prime}$ by $O[x] \cdot p$, we come back to a previous case, and the proof is complete.

COROLLARY. If O is an F-ring, then so is some quotient ring of ().

The foregoing theorem shows that if for some $n$ there exists a ring $O$ which is $n$-dimensional, while $O[x]$ is not $(n+1)$-dimensional, then there exist $F$-rings . Thus we may provisionally confine our attention to l-dimensional rings $O$.

THEOREM 4. If $O$ is 1-dimensional, and $O$ is a valuation ring, then $O[x]$ is 2-dimensional.

Proof. Let $p$ be a proper prime ideal of $O$, and let

$$
(0) \subset P \subseteq O[x] \cdot p \text {, }
$$

where $P$ is prime. Let

$$
f(x) \in P, \quad f(x) \neq 0 .
$$

Then one can factor out from $f(x)$ a coefficient of least value, that is, write

$$
f(x)=c \cdot g(x)
$$

where $c \in p$, and $g(x)$ has at least one coefficient equal to 1 ; in particular, then $g(x) \notin O[x]$. $p$; hence $c \in P$. So $P \cap O \neq(0)$, whence

$$
P \cap O=p \text { and } P=O[x] \cdot p \text {. }
$$

This proves that $O[x]$ is 2-dimensional ( see Corollary to Theorem 1 ).

Theorem 4 restricts the size of an $F$-ring, since a maximal ring is a valuation ring. The following theorem reduces the considerations to integrally closed rings.

THE OREM 5. Let $\bar{O}$ be the integral closure of the integral domain $O$. Then $O$ is an F-ring if and only if $\bar{O}$ is an F-ring. 
Proof. Let $R$ be an integral domain integrally dependent on $O$; a basic theoem of Krull ( see, for example, [2; Th. 4, p. 254]) says that if $P_{1} \subset P_{2}$ are prime ideals in $R$, then they contract to distinct prime ideals in $O$; hence $\operatorname{dim} R \leq \operatorname{dim} O$. Another theorem (loc. cit., p. 254) says that if $p_{1} \subset p_{2}$ are prime ideals in $O$, and $p_{1}$ is a prime ideal in $R$ contracting to $p_{1}$, then there exists a prime ideal $P_{2}$, $P_{2} \supset P_{1}$, contracting to $p_{2}$. Hence $\operatorname{dim} R \geq \operatorname{dim} O$, and so $\operatorname{dim} R=\operatorname{dim} O$. Hence $\bar{O}$ is 1 -dimensional if and only if $O$ is 1 -dimensional, and $\bar{O}[x]$ is 2-dimensional if and only if $O[x]$ is 2 -dimensional.

Thus if there exist $l$-rings, then there exist integrally closed $F$-rings, and, taking an appropriate quotient ring, we see that there would exist an integrally closed $F$-ring $O$ having just one proper prime ideal. In view of Theorem 4 (and the close association of integrally closed rings with valuation rings) one may ask whether an integrally closed ring with only one proper prime ideal is necessarily a valuation ring. Were it so, there would be no $F$-rings, but it is not so: Krull has an example [6; p.670f]. Wor convenience, we may mention the example: let $K$ be an algebraically closed field, $x$ and $y$ indeterminates; $O$ consists of the rational functions $r(x, y)$ which, when written in lowest terms, have denominators not divisible by $x$, and which are such that $r(0, y) \in K$.

3. Principal results. We now establish:

THEOREM 6. If $O$ is integrally closed with only one maximal ideal p, a an element of the quotient field of $O$, and $1 / a \notin O$, then $O[\alpha] \cdot p$ is prime. If also $\alpha \notin O$, then $O[a] \cdot p$ is not maximal.

Proof. We first observe that

$$
(O[\alpha] \cdot p, \alpha) \neq(1)
$$

as an equation

$$
1=c_{0}+c_{1} \alpha+\cdots+c_{s} \alpha^{s} \quad\left(c_{0} \in p, c_{i} \in O\right),
$$

leads to an equation of integral dependence for $1 / \alpha$ over $O$. Let now $g(x) \in$ $O[x]$ be a monic polynomial of positive degree. We may assume, trivially, that $a \notin O$; then $g(a)=c \in O$ is impossible, as $g(\alpha)-c=0$ would be an equation of integral dependence for $a$ over $O$; in particular, $g(\alpha) \neq 0$. Also $1 / g(\alpha) \notin$ $O$, for if it were in $O$, it would be a nonunit in $O$, and hence would be in $p$, so that

$$
1 \in g(\alpha) \cdot p \subseteq O[\alpha] \cdot p
$$

and this is not so. By the result on $\alpha$, 


$$
(O[g(\alpha)] \cdot p, g(\alpha)) \neq(1) .
$$

Since $\alpha$ satisfies $g(x)-g(\alpha)=0, O[\alpha]$ is integral over $O[g(\alpha)]$; over any prime ideal in $O[g(\alpha)]$ containing $(O[g(\alpha)] \cdot p, g(\alpha))$, there lies a prime ideal in $O[\alpha]$, hence

$$
(O[\alpha] \cdot p, g(\alpha)) \neq(1) .
$$

Since $1+g(x)$ is monic of positive degree, also

$$
(O[\alpha] \cdot p, 1+g(\alpha)) \neq(1) .
$$

This shows that $g(\alpha) \notin O[\alpha] \cdot p$, a conclusion that also holds if $g(x)$ is of degree zero; that is, $g(x)=1$.

We now prove that under the homomorphism $g(x) \rightarrow g(\alpha)$ of $O[x]$ onto $O[\alpha]$, the inverse image of $O[\alpha] \cdot p$ is $O[x] \cdot p$; this will complete the proof, as $O[x] \cdot p$ is prime but not maximal. Let, then,

$$
g(x) \in O[x], g(x) \notin O[x] \cdot p .
$$

We write

$$
g(x)=g_{1}(x)+g_{2}(x),
$$

where $g_{2}(x) \in O[x] \cdot p$ and no coefficient of $g_{1}(x)$ is in $p$; in particular, this is so for the leading coefficient $c$. Then $g_{1}(\alpha) / c \notin O[\alpha] \cdot p$, since $g_{1}(x) / c$ is monic. A fortiori, $g_{1}(\alpha) \notin O[\alpha] \cdot p$, whence also $g(\alpha) \notin O[\alpha] \cdot p$.

Corollary. In the case $\alpha \notin O$, if $g(x) \in O[x]$ and $g(\alpha) \in O[\alpha] \cdot p$, then $g(x) \in O[x] \cdot p$.

THE OREM 7. Let $O$ be an integrally closed integral domain, $p$ a proper ideal therein, $a$ an element in the quotient-field of $O$, but a $\notin O_{p}, 1 / a \notin O_{p}$. Then $O[a] \cdot p$ is prime but not maximal; in fact,

$$
O[\alpha] \cdot p \cap O=p \text { and } O[\alpha] / O[\alpha] \cdot p \simeq O / p[x] \text {. }
$$

Proof. We know that $O_{p}[\alpha] \cdot p$ is prime, and

$$
O_{p}[\alpha] \cdot p \cap O[\alpha]=O[\alpha]=O[\alpha] \cdot p
$$

by the last corollary (and the fact that $O_{p} \cdot p \cap O=p$ ). Hence $O[\alpha] \cdot p$ is prime. Also here, as in the corollary, we have that if $g(x) \in O[x]$ and $g(\alpha) \in O[\alpha]$. $p$, then $g(x) \in O[x] \cdot p$; the required isomorphism follows at once. 
Theorem 7 is known in the case that $O$ is a finite discrete principal order [3, $\$ 49$, p. 134-136]. The class of rings dealt with in the theorem includes this class properly; for example, the ring $O$ of the example of Krull is not a finite discrete principal order, as $x y^{\rho} \in O$ for all $\rho$, but $\gamma \notin O$.

THE OREM 8. If $O$ is 1-dimensional, then $O[x]$ is 2-dimensional if and only if every quotient ring of the integral closure of $O$ is a valuation ring.

Proof. By Theorem 5, we may assume $O$ to be integrally closed. If $O$ is an $F$-ring, then so is one of its quotient rings (Theorem 3, Corollary). This quotient ring is not a valuation ring (Theorem 4). Conversely, suppose some quotient ring $O_{1}=O_{p}$ is not a valuation ring. Let $\alpha$ be an element of the quotient field of $O_{1}$ such that $\alpha \notin O_{1}$ and $\alpha^{-1} \nsubseteq O_{1}$. Then $O_{1}[\alpha]$ is at least 2-dimensional, by Theorem 6 , and $O_{1}[x]$ is at least 3 -dimensional, as one sees by considering the homomorphism of $O_{1}[x]$ onto $O_{1}[\alpha]$ determined by mapping $x$ into $\alpha$. So $O_{1}$ is an $F$-ring. Thus $O_{p}[x] \cdot p$ is not minimal in $O_{p}[x]$, and it follows at once that $O[x] \cdot p$ is not minimal in $O[x]$, whence $O$ is an $F$-ring.

Let $O$ be the ring of Krull's example above, and let $X$ be an indeterminate. The single prime ideal $p$ in $O$ is constituted by the rational fractions $r(x, y)$ which, when written in lowest terms, have numerator divisible by $x$, i. e., are of the form $x g(x, y)$, where $g(x, y) \in K[x, y]$. The polynomials in $O[X]$ which vanish for $X=y$ form a prime ideal, different from $(0)$ since $x X-x y$ is in it, properly contained in $O[X] \cdot p$.

The following theorem is well known [4, Th. 13, p.376].

THE OREM 9. If $O$ is a Noetherian ring of dimension $n$, then $O[x]$ is $(n+1)$ dimensional.

Proof. Taking a quotient ring or residue class does not destroy the Noetherian character of $O$, so by Theorem 3 we may suppose $O$ is 1 -dimensional. Let then $p$ be a proper prime ideal in $O$. Then $O[x] \cdot p$ is minimal for every principal ideal $O[x] \cdot(a)$, where $a \in p, a \neq 0$, so by the Principal Ideal Theorem [3, p. 37], $O[x] \cdot p$ is minimal in $O[x]$, and $O[x]$ is 2-dimensional by Theorem 1 , Corollary. - Instead of the Principal Ideal Theorem, one could use instead that the integral closure $\bar{O}$ is also Noetherian (see, for example, [1, Th. 3, p.29]; see also [3, $\$ 39$, p. 108]). Neither proof makes use of the full force of the quoted theorems, so it might be of some interest to find a direct proof using less technical means.

NoTE. In a forthcoming paper we will show that if $O$ is a 1 -dimensional ring 
such that $O[x]$ is 2-dimensional, then $O\left[x_{1}, \cdots, x_{n}\right]$ is $(n+1)$-dimensional. Theoren 2 , above, will also be completed by examples showing that for any $m, n$ with $n+1 \leq m \leq 2 n+1$, there exist $n$-dimensional rings such that $O[x]$ is $m$ dimensional.

\section{ßEFERENCES}

1. I. S. Cohen, Commutative rings with restricted minimum condition, Duke Math. J. 17 (1950), 27-42.

2. I. S. Cohen and A. Seidenberg, Prime ideals and integral dependence, Bull. Amer. Math. Soc. 52 (1946), 252-261.

3. W. Krull, Idealtheorie, Ergebnisse der Mathematik und ihrer Grenzgebiete, 4, no.3, Berlin, 1935.

4. - Jacobsonsche Ringe, Hilbertscher Nullstellensatz, Dimensionstheorie, Math. Z. 54 (1951), 354-387.

5. - Beiträge zur Arithmetik kommutativer Integritätsbereiche, I. ivultiplikationsringe, ausgezeichnete Idealsysteme, Kroneckersche Funktionalringe. Math. Z. 11 (1936), 545-577.

6. - B'eiträge zur Arithmetik kommutativer Integritätsbereiche, II. v-Ideale und vollständig ganz abgeschlossene Integritätsbereiche. Math. Z. 41 (1936), 665-679.

University of California, Berkeley. 


\section{PACIFIC JOURNAL OF MATHEMATICS}

\section{EDITORS}

\section{R. U. FOEINSON}

University of California

Berkeley 4, California

E. HewitT

University of Washington

Seattle 5 , Washington
P. P. DILWOR TH

California Institute of Technology

Pasadena 4, California

E. F. BECKENBACH

University of California

Los Angeles 24, California

\section{ASSOCIATE EDITORS}

$\begin{array}{llll}\text { H. BUSEMANN } & \text { P. R. HALMOS } & \text { BØRGE JESSEN } & \text { J. J. STOKER } \\ \text { HERBERT FFDERER } & \text { IIEINZ HOPF } & \text { PAUL LÉVY } & \text { E. G. STRAUS } \\ \text { MARSHALL, IIALI } & \text { R. D. JAMES } & \text { GEORGE PÓLYA } & \text { KÖSAKU YOSIDA }\end{array}$

\section{SPONSORS}

UNIVERSITY OF BRITISH COLUMBIA

CALIFORNIA INSTITUTE OF TECHNOLOGY.

UNIVERSITY OF CAIJIFORNIA, BERKELEY

UNIVERSITY OF CAIJIFORNIA, DAVIS

UNIVERSITY OF CALIFORNIA, LOS ANGELES

UNIVERSITY OF CALIFORNI A, SANTA BARBARA

UNIVERSITY OF NEVADA

OREGON STATE COLLEGE

UNIVERSITY OF OREGON

\author{
UNIVERSITY OF SOU TIERN CALIFORNIA \\ STANFORD RESEARCH INSTITUTE \\ STANFORD UNIVERSITY \\ WASHINGTON STATE COLLEGE \\ UNIVERSITY OF WASHINGTON \\ AMERICAN MATHEMATICAL SOCIETY \\ NATIONAL BUREAU OF STANDARDS, \\ INSTITUTE FOR NUMERICAL ANALYSIS
}

Mathematical papers intended for publication in the Pacific Journal of Mathematics should be typewritten (double spaced), and the author should keep a complete copy. Manuscripts may be sent to any of the editors except Robinson, whose term expires with the completion of the present volume; they might also be sent to M.M. Schiffer, Stanford University, Stanford, California, who is succeeding Robinson. All other communications to the editors should be addressed to the managing editor, E. F. Beckenbach, at the address given above.

Authors are entitled to receive 100 free reprints of their published papers and may obtain additional copies at cost.

The Pacific Journal of Mathematics is published quarterly, in March, June, September, and December. The price per volume (4 numbers) is $\$ 8.00$; single issues, $\$ 2.50$. Special price to individual faculty members of supporting institutions and to individual members of the American Mathematical Society: $\$ 4.00$ per volume; single issues, $\$ 1.25$.

Subscriptions, orders for back numbers, and changes of address should be sent to the publishers, University of California Press, Berkeley 4, California.

Printed at Ann Arbor, Michigan. Entered as second class matter at the Post Office, Berkeley, California.

\section{UNIVERSITY OF CALIFORNIA PRESS • BERKELEY AND LOS ANGELES}




\section{Pacific Journal of Mathematics}

\section{Vol. 3, No. 2 \\ April, 1953}

William George Bade, An operational calculus for operators with spectrum

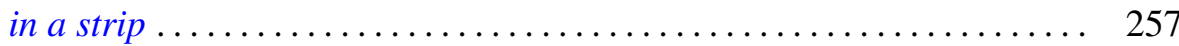

E. F. Beckenbach and Lloyd Kenneth Jackson, Subfunctions of several variables ..................................... 291

David Blackwell, Extension of a renewal theorem ................. 315

L. Carlitz, Some theorems on the Schur derivative ................ 321

Paul Arnold Clement, Generalized convexity and surfaces of negative curvature..................................... 333

Merrill M. Flood, On the Hitchcock distribution problem ............... 369

Watson Bryan Fulks, On the unique determination of solutions of the heat

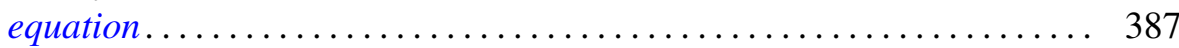

John W. Green, Length and area of a convex curve under affine transformation .................................... 393

William Gustin, An isoperimetric minimax .................. 403

Arthur Eugene Livingston, Some Hausdorff means which exhibit the Gibbs'

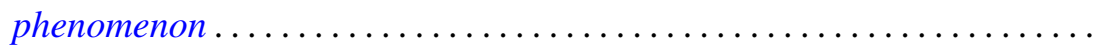

Charles Loewner, On generation of solutions of the biharmonic equation in the plane by conformal mappings ..................... 417

Gábor Szegő, Remark on the preceding paper of Charles Loewner ....... 437

Imanuel Marx and G. Piranian, Lipschitz functions of continuous

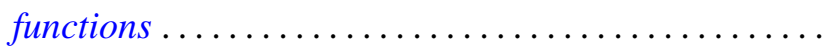

Ting-Kwan Pan, The spherical curvature of a hypersurface in Euclidean space ..

Ruth Lind Potter, On self-adjoint differential equations of second order ...

E. H. Rothe, A note on the Banach spaces of Calkin and Morrey...

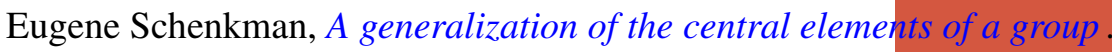

A. Seidenberg, A note on the dimension theory of rings .. . . 